\title{
On Laws of Nature
}

\section{Yiwei Yu}

DONGFENG COMMERCIAL VEHICLE Co., Ltd, Wuhan 430056, Hubei, China.

Abstract: Natural laws are at the heart of contemporary academic studies. Yet, the basic question on what is accounted as a law is still open to debate among philosophers of science. This paper provides a survey on three representativephilosophical accounts of laws of nature — the regularity (Humean) account, the necessitarian account by David Armstrong and the best system account of laws by David Lewis. By pointing out the disputes among these views whichstem from the dilemma pointed out by van Frassen - the tension between the problem of inference and the problem of identification, this paper provides a clear way to compare these accounts and a guidance for further research.

Keywords: David Armstrong; David Lewis; Laws of Nature

\section{Introduction}

To find the laws of nature is a well-motivated goal for human beings who have been observing and were attracted to patterns of the natural world for the past thousands of years. Natural science has thus far played a major role in decoding these patterns. From separate objects and incidents such as moving billiard balls and falling apples, natural scientists summarize regular patterns in their trajectories and derive law-like statements such as Newton's Second Law of Motion. These overarching patterns become increasingly comprehensive and accurate over the years. For some people, the laws of nature are nothing more than statements that summarize all the de-facto accidents in the world. This particular viewpoint is known as the regularity account or Humean account of laws. For others, the laws of nature bear necessitarian power which governs the events happening in this world. Just like how people must follow a certain code of conduct when legal laws are enforced, the laws of physics force things to behave in certain ways. This viewpoint is known as the necessitarian account of laws.

In this paper, we will be looking closely at the development of these two branches of theories. The paper consists of three main parts. In section two, I will explain the Regularity Account and analyze its problems. In section three, I will explain the Necessitarian Account and analyze its merits and problems compared with the regularity account. In section four, I will discuss a more sophisticated Humean account proposed by David Lewis — the Best System Account. Section five is the conclusion.

\section{Regularity account}

The regularity view of laws states that laws of nature are simply regularities or uniformities in the world and laws of nature are nothing more than statements that summarize all the de-facto accidents. This implies that there is no necessary connection between events around the world. For example, when we say "all copper conducts electricity" is a law, it simply means all copper is observed to be conductive, and no metaphysical inference could be made from the law. The idea of regularity view of laws is derived from Hume's skepticism against any empirical evidence for "necessity" between particular instances. This notion of necessity, "is nothing but an internal impression of the mind" (Hume 1896). In Hume's perspective, the belief that the natural laws necessitate the facts is due to our psychological tendency to link two events that happen successively. Laws are informative and concise summaries of natural regularities, in which certain types of instances are followed by some other

Copyright(C) 2020 Yiwei Yu

doi: 10.18686/ahe.v4i10.2935

This is an open-access article distributed under the terms of the Creative Commons Attribution Non-Commercial License (http://creativecommons. org/licenses/by-nc/4.0/), which permits unrestricted non-commercial use, distribution, and reproduction in any medium, provided the original work is properly cited. 
types of instances. There is no metaphysical distinction between law-like statements and true propositions. To better understand this, we can simply imagine two worlds A and B, in which all facts appear to be the same. However, while A is law-governed, $\mathrm{B}$ is not. If the law "all copper conducts electricity" is true for both A and B, it means all copper in A is necessarily conductive, and all copper in $\mathrm{B}$ is de-facto conductive. Humean theorists claim that there is no way for us to distinguish between the two worlds. (Elder 1994)

Regularity view of laws is one of the most basic accounts of laws of nature. However, it is subject to certain limitations. In this section, we will be looking at four of them closely: (1) Regularities are not sufficient to be laws (2) Regularities are not necessarily laws (3) The problem of Inference and (4) The problem of Circular explanation.

First of all, regularities are not sufficient to be laws, and the regularity view may mistake some accidental generalizations to be laws. For example, from the regularity view, both (1) "No pure gold sphere is heavier than 1000kg" and (2)"No pure uranium sphere is heavier than 1000kg" are true generalizations of our world and thus both should be regarded as a law of nature. (van Fraassen 2003) However, there are differences between these two statements because "No pure gold sphere is heavier than 1000kg" is just an accident, and we can actually make a gold sphere larger than 1000kg if we decide to do so. Making a $1000 \mathrm{~kg}$ uranium sphere, on the other hand, is impossible, as the critical mass of uranium is limited at $57 \mathrm{~kg}$. Therefore, this account can fail in distinguishing true laws from mere regularities.

Besides, some laws are not necessarily regularities. The regularity view also fails to account for laws that are true but may not have actual instances. For example, "objects moving in a straight line with constant velocity will continue their motion forever on an absolutely smooth surface" is hardly qualified as a law under the regularity view. There is no absolutely smooth surface in the world. Thus, there is no strictly de-facto generalizations to make. If we allow hypothetical generalizations to be characterized as laws, then we still face difficulties in distinguishing laws and non-laws in hypothetical circumstances. Both statements "all unicorns have wings" and "objects moving in a straight line with constant velocity will continue their motion forever on an absolutely smooth surface" are (argubly) true hypothetical generalizations, but the regularity account does not provide a manifest justification for denying the former while accepting the latter as a law.

Next, under the regularity account, laws lack the inferential power. Generally speaking, we can infer facts from laws it is a law that $\mathrm{A}$ implies $\mathrm{A}$. The regularity account does not explain how the regularities arise and denies any necessitation between laws and facts, which undermines the inferential power of regularist laws. By denying that there is a deeper reason as to why the patterns exist, the regularity view has to admit it just happens to be that we live in a world with patterns. However, if we, by chance, live in a world that has patterns, we cannot be sure that these patterns will persist. For example, a teacher has a certain confidence that the same group of people will still be present at school tomorrow because she knows that coming to school is compulsory for them. On the contrary, if coming to school is not necessary nor compulsory, the teacher would not be able to infer that the students will still be in school the next day. If we do not believe that there is a deeper connection between the patterns in the world, we would not have good reasons to believe that they will persist in the future. It would seem contradictory if we believe laws are mere regularities, but at the same time make inferences based on the belief that the patterns of our world will continue to exist.

Another concern for the regularity view is its circularity in explanation. If we extract laws by summarizing the facts and use the laws to explain the facts, this seems to be a form of self-validation instead of a fundamental explanation. For instance, from observations, we know that the north pole of a magnet always attracts the south pole but repels another north pole. From here, we generalize this regularity into a law that states all like magnetic poles repel and unlike magnetic poles attract. However, when we need to explain why one side of a bar magnet attracts one side of another bar magnet but repels the other side of it, we quote the previous generalization to explain this phenomenon. Therefore, it is reasonable for us to doubt whether these law-like statements in the regularity view is sufficient for bridging the conceptual distance between explanans and explananda. (Hempel 1991)

The regularity view, upon analyzing, is not good enough to account for the laws of nature. It would be hasty to generalize all regularities to be laws as it is evident that there are more special traits to laws than mere regularities. To this end, both David Armstrong and David Lewis have given their attempts to identify these traits.

\section{Necessitarian account}

The necessitarian account of laws identifies the laws of nature as underlying principles that govern the facts in the world. On the necessitarian view, the laws of nature bear physical (nomological) necessity which is not as strong as logical necessity 
but stronger than mere regularities. On the regularity account, "apples fall from trees" is a law because all apples fall from trees. However, on a necessitarian view, all apples must fall from trees because it is a law that "apples fall from trees". This concept of physical necessity is most famously explained by David Armstrong in his Laws of Nature As Relationships Between Universals, and As Universals:

(a) It is a law that Fs are Gs

cannot be reduced to

(b) Each $\mathrm{F}$ is a G.

(Armstrong 1982)

Armstrong believes that the properties of F and G (i.e. F-ness and G-ness) are two universals, and the relationships between F-ness and G-ness is also a kind of universal. This third kind of universal is what he regards as physical necessities, manifested in (a) but not (b). Armstrong believes statement (a) cannot be reduced to statement (b). The two statements are not equal metaphysically and the derivation is asymmetric. We can conclude from "it is a law that Fs are Gs" that "All Fs are Gs", while from "Each $\mathrm{F}$ is a G" we cannot draw the conclusion that "it is a law that Fs are Gs".

The idea is well-motivated by our human intuition that laws of nature necessitate certain events to happen-when event $\mathrm{F}$ and $\mathrm{G}$ are prescribed to the relationship described in (a), then if $\mathrm{F}$ happens, G must follow. For instance, it is common to sense that when a net force is applied to an object, the object must accelerate accordingly, and nothing fails to obey that law of nature.

What is good about the necessitarian account is that it addresses the limitations of the regularity account. On the necessitarian views, bearing physical necessities is necessary and sufficient for statements to be laws. In this way, we can better distinguish laws from mere particulars, solving problem (1) and (2) faced by regularity view. For instance, we can now distinguish the gold and uranium statements mentioned above. "No pure gold sphere is heavier than 1000kg" is not a law as it is not physically necessary that a pure gold sphere should not be heavier than $1000 \mathrm{~kg}$. On the other hand, no pure uranium sphere can be heavier than $1000 \mathrm{~kg}$ is constrained by the physical necessity between uranium and its critical mass.

Moreover, the necessitarian view provides an answer to a question that Humeans struggle to answer: why do regularities exist? The necessitarian account attempts to solve this problem by granting laws a necessitarian power. Therefore, "a law that A" is equivalent to "It is necessary that A". For example, the necessitarian account equates "all copper conducts electricity" to "it is necessary that all copper conducts electricity." When A becomes a necessity, we are able to infer that A is the case. In this way, the law is strong enough for inferences. These physical necessities also provide us with more fundamental explanations to phenomena and a deeper reason why particular things happen, solving both problem (3) and (4) faced by regularity view.

However, by solving the problem of inference, the necessitarian view exposes itself to the problem of identification. When we say, "it is necessary that all copper conducts electricity", we need to at least explain where this necessity comes from and how it works. In order to answer these questions, we have to refer to some empirical facts as evidence. Yet, none of the empirical results is able to prove this necessitarian power. (van Fraaseen 2003) Scientific experiments and results only provide us with empirical observations of the world. The fact that all copper conducts electricity corresponds to the statement (b) all Fs are Gs. As Armstrong claimed, statement (a) cannot be reduced to statement (b) because they are not the same metaphysically. Our empirical evidence, which only allows us to infer statement (b), is not sufficient to establish statement (a). That is, scientific observations can only directly support our claims about regularities, but not our metaphysical commitment to the governing power of these regularities. Since we cannot deduce statement (a) from statement (b), we cannot declare the physical necessity through any empirical observations. Armstrong fails to clarify the identifiable aspect of nature that makes for necessitarian laws.

\section{Best system account}

David Lewis (1983) points out that Armstrong's necessitarian account seems to avoid the question instead of addressing it. By simply naming these restrictions "physical necessity", Armstrong answered neither what these restrictive forces are, nor where they come from. Lewis famously criticises Armstrong's approach as follows:

The mystery is somewhat hidden by Armstrong's terminology. He uses 'necessitates' as a name for the lawmaking universal $N$; and who would be surprised to hear that if $F$ 'necessitates' $G$ and has $F$, then a must have G? But I say that $N$ deserves the name of 'necessitation' only if, somehow, it really can enter into the requisite necessary connections. It can't enter into them just by bearing a name, any more than one can have mighty biceps just by being called 'Armstrong'.

(Lewis, 1983) 
Ultimately, we all know that there seem to be certain patterns that objects in the cosmos obey (locally), for example, the conservation laws. However, simply claiming there are physical necessities is not a proof of their existence, especially when no empirical evidence of physical necessities is obtainable, as argued in the previous section. In response to Armstrong's necessitarian view, Lewis adopts Hume's argument that the necessary causal relationships understood by humans may not actually exist. It is a psychological tendency for humans to link two consecutive events with necessitation-Because all past event $\mathrm{A}$ is followed by event $\mathrm{B}$, we believe that event $\mathrm{A}$ must be followed by event B. For instance, because we knew by experience that rigid bodies bounce off upon collision, we believe that a "force" must exert on billiard ball B and causes it to move away when hit by another rapidly moving billiard ball A.

Lewis' solution is to add a criterion for laws based on the regularity account. According to Lewis' original account, the laws of nature in a given possible world are the principles of the best scientific theory of that world, where "best" denotes an optimal combination of strength and simplicity. This is known as Lewis' "Best System Account" of laws. Lewis believes that all laws supervene on particulars. Supervenience refers to the relation between sets of properties or sets of facts.(Lewis, 1994) $\mathrm{X}$ is said to supervene on $\mathrm{Y}$ if and only if some difference in $\mathrm{Y}$ is necessary for any difference in $\mathrm{X}$ to be possible. In simpler words, this means laws cannot change without changes in particulars and only changes in particulars result in changes in laws. Therefore, he believes that the laws of nature should not be metaphysically over and above any particulars and agrees with the foundational principle of the regularitist approach.

How Lewis' Best System Account explains the laws is totally different from Armstrong's necessitarian view. Taking the aforementioned two billiard balls as an example. In physics, we explain it by quoting Newton's Second Law of motion, $\mathrm{F}=\mathrm{ma}$. Because the first billiard ball exerts a force on the second billiard ball, the second one accelerates and moves away. In Armstrong's view, the physical necessity makes the second ball to bounce off upon collision. F=ma is a law means when the first ball collides with the second, there must be a force being exerted and the second ball must accelerate with a net force exerted on it. However, Lewis would argue that $\mathrm{F}=\mathrm{ma}$ is only an axiom summarized from motions of all objects. Lewis also agrees that $\mathrm{F}=\mathrm{ma}$ is a law but the reason is that it is one of the simplest and strongest formulas that applies universally to all interactions among objects, including the collision of two billiard balls.

Lewis' account has ingeniously addressed the major flaws of the basic regularity view. If we consider all regularities to be laws, we would realize that, as we mentioned above, not all regularities are good enough to be laws. Under Lewis' criteria, only the simplest and strongest statements of regularities should be considered as the laws of nature.

For example, we can imagine ourselves asking the Laplace's Demon what the world is like. One way he could describe the world for us is to list out the motion and state of every particle, which would be a too exhaustive list which can go on forever. This statement is fairly strong as it provides the most accurate description of every unit in this world. However, due to our limited life span as human beings, we cannot follow this infinitely. Therefore, strong but complex statements like this are not suitable to be our natural laws. On the other hand, there are simple and true statements of regularities but contain too little information. For example, "I am alive" is a short and sweet statement but it does little to provide information about the natural world.

Hence, if we are to pick out the best statements that help us understand the natural world in the simplest way. We would choose those like $\mathrm{F}=\mathrm{ma}$ or Schrodinger equation that are strong yet simple_ _ given the initial condition, the simple equations of motion provide a tremendous amount of information about where particles would be.

However, even such criteria are appealing to most, they still seem rather vague and arbitrary. What exactly do we mean by simple and strong? Is the simplicity determined by the number of words in the statement, and in that case, what language should we use? Is the strength measured by the number of particulars it covers, but how do we quantify that? Although we do get the gist of these two words, they need a clearer and universal definition. Moreover, Lewis mentioned that laws of nature should be the best combination of simplicity and strength, but how do we balance simplicity and strength when long sentences contain more information and short sentences contain less in general? Lewis' account fails to show us the picture of clearly defined laws of nature due to the vagueness in its criteria. But we are not hopeless here. Lewis has demonstrated to us a good direction to go. Future philosophers of science can still work on and improve these criteria, and by modifying and refining these criteria, we can see ourselves getting closer and closer to the essence of laws of nature.

Moreover, many opponents of the best system accounts argue that the Best System Account and Humean approach to the laws of nature, in general, is too anthropocentric. Even if we assume that we've solved the vagueness problem in 
Lewis' account and have agreed upon a set of universal criteria for the laws of nature (assume the criteria are "simplest and strongest"), we can expect a group of aliens who think differently from us to develop a different set of "simplest and strongest" laws. “In Douglas Adams' Hitchhiker's Guide to the Galaxy, the supercomputer Deep Thought is tasked with computing the Ultimate Question of Life, the Universe and Everything. Famously, after 7.5 million years of computation, the computer reveals the answer, 42."(Craig 2020) This shows that whatever statements a group of aliens picked as simplest and strongest can be different from ours. In this way, laws are no longer objective and can vary from one perspective to another. Rather than laws of nature, it is now laws of nature for the human and limited to human understanding and interpretation.

This criticism against Best System Account is definitely valid but should not be as worrying as many may believe. In the end, we need to consider whether a set of absolutely objective laws are ever obtainable and whether it is necessary for laws to be absolutely objective. Even if one day an absolutely objective set of laws of nature is found through some super machine, it might just be a number 42 which can neither help us produce, create, predict nor make any scientific or technological progress.

\section{Conclusion}

In this paper, I have explained three main theses for the laws of nature: the regularity account, the necessitarian account and the best system account. However, none of the three accounts can provide a satisfactory solution thus far. There seems to be a tension between the Humean view (including both the regularity account and the best system account) and the necessitarian view. The tension is, as identified by van Fraassen (2003), between the problem of inference and the problem of identification.

These two problems together form a dilemma. There are two desiderata that cannot be simultaneously attained - the inferential power of laws which comes from their necessitations and the empirical accessibility of such necessitations. We seem to be standing at a crossroad deciding whether to take one step forward to make a metaphysical commitment allowing a law A to imply that A must be the case, or to take one step back and only focus on the empirical results we observed in the past which are the only identifiable aspects of the nature. There is a gap between what we can empirically access and whatpower we hope the laws to possess.

When metaphysical postulations are completely disjointed from the empirical evidence, it is tempting to forgo the metaphysical program and focus purely on empirically analyzable entities. Lewis' best system account is a good place to start. Carrying forward the identifiable aspects of laws from regularity account, the best system account grants laws a superior(nonmetaphysically) position compared to mere particulars. Laws are different from particulars because they are simple and informative. Although these criteria may need to be improved further, it is possible that future philosophers can work on detailing and refining these criteria or find an alternative non-Lewisian Humean account that solves the problem that Lewisian Best System Account faces.

\section{References}

1. Armstrong DM. What is a law of nature?. Cambridge: Cambridge University Press; 2016.

2. Armstrong DM. Laws of nature as relations between universals and as universals. Philosophical Topics 1982; 13(1): 7-24. doi: 10.5840/philtopics19821311.

3. Bas C, Van F. Laws and symmetry. Oxford: Clarendon Press; 2003.

4. Beebee H. Hume and the problem of causation. Oxford Handbooks Online; 2014. doi: 10.1093/oxfordhb/9780199742844.013.8.

5. Craig C. Humean laws of nature: The end of the good old days(Draft); 2020.

6. Elder CL, Laws, Natures, et al. Philosophy and phenomenological research 1994; 54(3), 649. doi: 10.2307/2108585.

7. Hempel C. Laws and their role in scientific explanation; 1991.

8. Hume D. A treatise of human nature 1896; Section 1.3.14.20.

9. Lewis D. New work for a theory of universals. Australasian Journal of Philosophy 1983; 61(4), 343-377. doi: 10.1080/00048408312341131.

10. Lewis D. Symposium: Chance and credence. Mind 1994; 103(412), 473-490. doi: 10.1093/mind/103.412.473. 